\title{
Aniridia-cerebellar ataxia-intellectual disability syndrome
}

INSERM

\section{Source}

INSERM. (1999). Orphanet: an online rare disease and orphan drug data base. Aniridiacerebellar ataxia-intellectual disability syndrome. ORPHA:1065

Aniridia-cerebellar ataxia-intellectual disability syndrome, also known as Gillespie syndrome, is a rare, congenital, neurological disorder characterized by the association of partial bilateral aniridia with non-progressive cerebellar ataxia, and intellectual disability. 